Research Article

Open Access

\title{
Improvement of defecation in healthy individuals with infrequent bowel movements through the ingestion of dried Mozuku powder: a randomized, double-blind, parallel-group study
}

\author{
Masaki Matayoshi $^{1}$, Junjiro Teruya ${ }^{1}$, Mina Yasumoto-Hirose ${ }^{2}$, Ryuji Teruya $^{3}$, Naoki Miura $^{4}$, \\ Ryuji Takeda 5
}

${ }^{1}$ Okinawa TLO Ltd. Nishihara Okinawa 903-0213 Japan, ${ }^{2}$ Tropical Technology Plus Uruma, Okinawa 904-2234, ${ }^{3}$ Okinawa Industry Promotion Public Corporation Naha, Okinawa 904-2161 Japan, ${ }^{4}$ Miura Clinic Osaka-city, Osaka 530-0044 Japan, Faculty of Health Sciences for Welfare, Department of Nutritional Sciences for Well-being, Kansai University of Welfare Sciences Kashiwara Osaka 582-0026 Japan.

*Corresponding Author: Ryuji Takeda PhD., Faculty of Health Sciences for Welfare, Department of Nutritional Sciences for Well-being, Kansai University of Welfare Sciences Asahigaoka 3-11-1, Kashiwara Osaka 582-0026, Japan.

Submission Date: March 31 ${ }^{\text {st }}$, 2017, Acceptance Date: September 27 ${ }^{\text {th }}$, 2017, Publication Date: September $30^{\text {th }}, 2017$

Citation: Matayoshi M., Teruya J., Yasumoto-Hirose M., Teruya R., Miura N., Takeda R., Improvement of Defecation in Healthy Individuals with Infrequent Bowel Movements Through the Ingestion of Dried Mozuku Powder: A Randomized, Double-blind, Parallel-group Study. Functional Foods in Health and Disease 2017; 7(9); 735-742.

https://doi.org/10.31989/ffhd.v7i9.350

\begin{abstract}
:
Background: Okinawa mozuku (Cladosiphon okamuranu) is a type of edible seaweed of the family Chordariaceae that typically contains the polysaccharide fucoidan as a functional ingredient. In Okinawa, raw mozuku is eaten as vinegared mozuku together with vinegar or as tempura (deep-fried in batter). Polysaccharides such as fucoidan are generally known to regulate intestinal function, which is why we have used Okinawa mozuku to investigate this intestinal regulatory effect.
\end{abstract}

Methods: The study was designed as a randomized, double-blind, parallel group study. Dried Okinawa mozuku powder at a dose of $2.4 \mathrm{~g} /$ day (1.0 g/day of fucoidan) and a placebo not 
containing any dried Okinawa mozuku powder were each made into capsules and given to healthy men and women with infrequent weekly bowel movements (2-4 movements a week) to ingest for eight weeks. We then investigated changes in the defecation situation, blood tests, and adverse events.

Results: In the group that ingested the capsules containing dried Okinawa mozuku powder, the number of days with a bowel movement significantly increased compared with the placebo group after four weeks of ingestion $(p<0.05)$. Furthermore, after eight weeks of ingestion, the same increasing trend was seen compared with the placebo group $(p=0.0964)$. The volume of stool also increased significantly in the dried Okinawa mozuku powder group after eight weeks compared with the placebo group. In terms of blood tests and adverse events, no adverse events occurred that were the result of the test food.

Conclusion: Ingestion of Okinawa mozuku was found to have a regulatory effect on intestinal function by promoting defecation in healthy individuals with a tendency for constipation. This demonstrated that Okinawa mozuku is a functional food capable of making defecation smoother and increasing the volume of stool.

Keywords: Okinawa mozuku, Cladosiphon okamuranus, fucoidan, dry powder, bowel movement, constipation

\section{BACKGROUND}

Okinawa mozuku (Cladosiphon okamuranus Tokida) is classified under the order Chordariales family, Chordariales genus, and cladosiphon. It is an edible seaweed that is native in areas spanning the Yaeyama islands in Okinawa prefecture to Amami-Oshima in Kagoshima prefecture [1]. Some of the reported benefits of Okinawa mozuku include its anticoagulant effects [2], lowering of LDL cholesterol [3], anti-HIV-virus activity [4] , improvement of functional gastrointestinal damage[5], and increasing intestinal vermiculation [6].

The components involved in these benefits of Okinawa mozuku are believed to be the highmolecular polysaccharides. Among these, fucoidan is particularly well known in addition to the chemical structure of fucose as the constituent sugar, with a side chain of uronic acid and sulfate group [7].

Constipation is an abdominal symptom that is generally due to lack of fiber. Decreased vermiculation of the intestines makes it difficult to have regular bowel movements. When long periods of constipation continue, the stool loses its water content due to staying in the intestines 
for too long, which causes it to harden. Furthermore, chronic retention of stool in the intestines causes putrid bacteria to increase in the intestinal flora, thereby reducing the number of lactobacillus. This generates indole, skatole, ammonia, and hydrogen sulfide in the intestines, which causes unpleasant symptoms such as infection of the intestinal tissues and feeling bloated. Constipation is not only associated with abdominal symptoms but can also cause psychological symptoms [8-10]. Abdominal bloating lowers appetite and leads to feelings of depression. Consequently, it is clear that regular bowel movements are essential for maintaining a high quality of life. In order to improve the bowel routine, it is important to increase the amount of fiber intake in order to increase lactobacillus or bifidus in the intestinal flora and to lower the emission of putrefaction gases [11]. For example, fucoidan extracted from the Okinawa mozuku has also been reported to improve constipation. However, these are reports which have been based on the intake of refined fucoidan only. Moreover, this is the first report on improvement of the bowel routine from taking Okinawa mozuku by itself. Therefore, we conducted a placebo-controlled double-blind test to study the bowel movement improvement effects of dried Okinawa mozuku.

\section{METHODS}

\section{Study design}

This study was conducted as a randomized, double-blind, parallel group study. Thirty individuals participated in the study (15 in the Okinawa mozuku group and 15 in the placebo group). The study participants were all healthy adults with infrequent bowel movements (2-4 movements a week) who met all of the inclusion criteria and none of the exclusion criteria listed in Table 1. All the participants gave their informed consent to take part in the study. The Western Ethics Committee reviewed and approved the study prior to its implementation. Additionally, the study plan was registered with the UMIN Clinical Trials Registry prior to being conducted. (UMIN000025173)

Table 1. Inclusion and exclusion criteria used screening subjects for the study

Inclusion criteria

1. Subject must be $>20$ years of age

2. Infrequent bowel movements (2-4 movements a week)

Exclusion criteria

1. Known allergy or sensitivity for any foods

2. Subjects who uses any medecines or supplements

3. Histry of severe disease like intestinal disease 


\section{Randomization}

Individuals with no problem participating in the study were allocated to one of two groups by the stratified block randomization method (stratification factors: number of bowel movements and age). An independent person responsible for allocation not involved in the study created a computer program using a random number table to allocate the participants into one of two groups. Both the participants and the investigators were blinded to the group allocations.

\section{Placebo and test foods}

The test food was in the form of capsules containing Okinawa mozuku. The dose was $2.4 \mathrm{~g} / \mathrm{day}$ (1.0 g/day of fucoidan). Meanwhile, the placebo food was a capsule containing starch instead of Okinawa mozuku. The two foods had no differences in terms of appearance including color, smell, and size. But there was a difference in salty taste in the capsule. The nutritional components and compositions are presented in Table 2.

Table 2. Nutritional composition of the test foods and placebo * $(2.4 \mathrm{~g}$ capsels/day $)$

\begin{tabular}{lcc}
\hline & $\begin{array}{c}\text { Okinawa mozuku } \\
\text { (Test Food) }\end{array}$ & Placebo \\
\hline Energy (kcal) & 3.6 & 7.9 \\
Protein (g) & 0.1900 & 0.002 \\
Lipid (g) & 0.1000 & 0.002 \\
Carbohydrates (g) & 1.50 & 1.95 \\
Sodium (mg) & 45.600 & 0.004 \\
Fucoidan (g) & 1.0 & 0.0 \\
\hline
\end{tabular}

\section{Bowel movement condition (investigation of defecation)}

Throughout the study period, the participants answered questionnaires on fecal properties, including the number of bowel movements per day, the number of bowel movements per week, the volume of stool per day (number of medium “egg"-sized stools), and the hardness of stool each day (hard to watery). The questionnaires were tabulated every four weeks.

\section{Blood analysis and adverse events}

Hematological and blood biochemical tests were completed before the start of ingestion, after four weeks of ingestion, and after eight weeks of ingestion. Participants were also interviewed about adverse events at each hospital visit to investigate causal relationships and severity. 


\section{Statistical analysis}

The Mann-Whitney U test and Wilcoxon signed-rank test were performed to examine the number of days with a bowel movement, the number of bowel movements, the hardness of stool, and the volume of stool. The level of statistical significance was set at $5 \%$ and a $p$-value of $\geq 5 \%$ to $<10 \%$ was considered to indicate a difference in trend. Blood tests were compared between groups by using Student's $t$-tests. Adverse events were examined using Fisher's exact test. Statistical analyses were done at Kansai University of Welfare Sciences using the software SAS ver. 9.4.

\section{RESULTS}

\section{Analysis set}

The study included a total of 30 participants, with 15 subjects in each group. No participants dropped out half way through the study or discontinued the study. The analysis set for efficacy and safety therefore included all the participants. Table 3 shows the profile of subjects who participated in the study

Table 3. Profile of Participated subjects in the study

\begin{tabular}{lccc}
\hline & Okinawa mozuku & Placebo & p-value \\
\hline Number & 15 & 15 & - \\
Gender Ratio (male/female) & $11 / 4$ & $11 / 4$ & $1.000^{* 3}$ \\
Age & $45.1 \pm 10.0$ & $45.1 \pm 11.0$ & $1.000^{* 3}$ \\
Number of bowel movements per day & $0.40 \pm 0.11$ & $0.38 \pm 0.99$ & $0.4939^{* 4}$ \\
Number of days with a bowel & & & \\
movement & $2.70 \pm 0.73$ & $2.53 \pm 0.55$ & $0.6180^{* 4}$ \\
( day/week) & & & \\
Volume of stool ${ }^{* 1}$ (number/day) & $5.99 \pm 1.75$ & $5.84 \pm 1.22$ & $0.5473^{* 4}$ \\
Hardness of stool ${ }^{* 2}$ & $2.48 \pm 1.00$ & $2.21 \pm 0.75$ & $0.8682^{* 4}$ \\
\hline${ }^{* 1}$ number of medium "egg”-sized stools, ${ }^{* 2}$ 2score 1 (hard) to 5 (watery) & \\
${ }^{* 3}$ Fisher's exactry test, ${ }^{* 4}$ Student's t test, ${ }^{* 5}$ Mann-Whitney U test & &
\end{tabular}

\section{Bowel movement condition}

The results of the study are presented in Table 4. The number of bowel movements per day rose significantly in both groups compared with before ingestion, although no difference was seen between the groups. Meanwhile, the number of days with a bowel movement per week increased significantly in the Okinawa mozuku group compared with the placebo group after four weeks of 
ingesting the test food $(\mathrm{p}=0.0438)$. After eight weeks of ingestion, the Okinawa mozuku group showed a significantly increasing trend compared with the placebo group $(p=0.0964)$. The volume of stool per day also increased significantly in the Okinawa mozuku group compared with the placebo group. After eight weeks of ingestion the hardness of stool had significantly softened compared with before ingestion, but did not differ between the two groups.

Table 4. Fecal Properties: Before, 4-week and 8-week

\begin{tabular}{|c|c|c|c|c|c|c|c|c|c|c|c|}
\hline & Group & $\mathrm{n}$ & \multicolumn{3}{|c|}{ Before } & \multicolumn{3}{|c|}{4 week } & \multicolumn{3}{|c|}{8 week } \\
\hline $\begin{array}{l}\text { Number of bowel } \\
\text { movements per day }\end{array}$ & $\begin{array}{l}\text { Okinawa } \\
\text { mozuku }\end{array}$ & 15 & 0.40 & \pm & 0.11 & 0.71 & \pm & $0.25^{*}$ & 0.76 & \pm & $0.30^{*}$ \\
\hline (number/day) & Placebo & 15 & 0.38 & \pm & 0.09 & 0.67 & \pm & $0.47^{*}$ & 0.70 & \pm & $0.52^{*}$ \\
\hline $\begin{array}{l}\text { Number of days with a } \\
\text { bowel movement }\end{array}$ & $\begin{array}{l}\text { Okinawa } \\
\text { mozuku }\end{array}$ & 15 & 2.70 & \pm & 0.73 & 4.47 & \pm & $1.33^{* \#}$ & 4.60 & \pm & $1.49^{\$ \dagger}$ \\
\hline ( day/week) & Placebo & 15 & 2.53 & \pm & 0.55 & 3.57 & \pm & $1.46^{*}$ & 3.73 & \pm & $1.62^{*}$ \\
\hline Hardness of stool & $\begin{array}{l}\text { Okinawa } \\
\text { mozuku }\end{array}$ & 15 & 2.48 & \pm & 1.00 & 3.30 & \pm & $0.69^{*}$ & 3.78 & \pm & $0.66^{*}$ \\
\hline $\begin{array}{l}\text { (Score } 1 \text { (hard) to } 5 \\
\text { (watery)) }\end{array}$ & Placebo & 15 & 2.21 & \pm & 0.75 & 3.13 & \pm & $0.88^{*}$ & 3.33 & \pm & $1.11^{*}$ \\
\hline Volume of stool & $\begin{array}{l}\text { Okinawa } \\
\text { mozuku }\end{array}$ & 15 & 5.99 & \pm & 2.45 & 6.68 & \pm & 1.75 & 7.68 & \pm & $1.93^{* \#}$ \\
\hline $\begin{array}{l}\text { (number of medium "egg"- } \\
\text { sized stools) }\end{array}$ & Placebo & 15 & 5.84 & \pm & 1.92 & 6.76 & \pm & $2.11^{*}$ & 6.16 & \pm & 2.36 \\
\hline $\begin{array}{c}* \mathrm{p}<0.05 \text { Wilcoxon signed- } \\
\text { rank test vs } 0 \text { week }\end{array}$ & $\begin{array}{l}{ }^{\$} \mathrm{p}<0.10 \mathrm{Wi} \\
\text { rank test vs }\end{array}$ & wee & & & & & & & & & \\
\hline $\begin{array}{l}{ }^{\#} \mathrm{p}<0.05 \text { Mann-Whitney } \mathrm{U} \\
\text { test vs placebo group }\end{array}$ & $\begin{array}{l}{ }^{\dagger} \mathrm{p}<0.10 \mathrm{Mc} \\
\text { test vs plac }\end{array}$ & $-\mathrm{H}$ & ey U & & & & & & & & \\
\hline
\end{tabular}

\section{Blood Analysis and Adverse Events}

No changes that could be medically problematic were observed in the blood test results of either group (data not shown). Moreover, while mild adverse events such as bloating occurred, these did not differ significantly between the groups.

\section{DISCUSSION}

We had subjects take $2.4 \mathrm{~g}$ (fucoidan:1.0 g ) of Okinawa mozuku to examine its effects on improving bowel movements. We found that four weeks of taking this preparation resulted in significantly increased bowel movement frequency compared to the control group. In past research, 
Tomori et al [12] reported that taking fucoidan extracted from Okinawa mozuku at $0.5 \mathrm{~g} / \mathrm{day}$ improved the bowel habits of somewhat constipated healthy individuals. We also found in our study that taking the powder of dried mozuku by itself had the same effects on improving bowel habits. Improvements in gastrointestinal symptoms were reported in past research by Miyoshi et al. [6], who found that taking fucoidan extracted from Okinawa mozuku $810 \mathrm{mg} /$ day for 4 weeks promoted vermiculation of the intestines. In all past reported cases, refined fucoidan from Okinawa mozuku was taken, and the fucoidan amount was between $0.5 \mathrm{~g}-0.81 \mathrm{~g}$. We demonstrated that taking dried mozuku by itself had the same effects on improving bowel movements as taking refined fucoidan. The results of this trial showed that taking Okinawa mozuku directly also regulated intestinal function. Thus, as past reports on improvements in bowel movements from taking refined fucoidan in Okinawa mozuku resulted in almost the same result of improvement, we believe that the fucoidan contained in Okinawa mozuku is the functional component that regulates bowel activity. These mechanisms are not clear, but Ihara reported that fucoidan enhanced intestinal epithelial barrier function by upregulating the expression of claudin-1 [13]. As a result, we think that fucoidan may be an appropriate therapy for the treatment of inflammatory bowel diseases.

\section{CONCLUSION}

We conducted a randomized, double-blind, parallel group study using capsules containing dried Okinawa mozuku powder. We found that in the Okinawa mozuku group, the number of days with a bowel movement per week increased and the volume of stool per day significantly increased compared with the placebo group. The aforementioned results demonstrate that Okinawa mozuku is a functional food with a regulatory effect on intestinal function.

Competing Interests: The authors have no conflicts of interest directly relevant to the content of this article. This study was carried out consignment of Okinawa Prefecture, Japan.

Authors' Contributions: M Matayoshi, J Teruya, R Teruya, Y Hirose and R Takeda designed the study; N. Miura performed the practical part of the study, in addition to the statistical analysis of the data with supervision from R Takeda; R Takeda, M Matayoshi, Y Hirose drafted the manuscript and all authors engaged in the manuscript work. All authors have read and approved the final manuscript.

Acknowledgements and Funding: This study was carried out in consignment of Okinawa Prefecture, Japan. 


\section{REFERENCES}

1. Shinmura I, Yamanaka K: Studies on the Cultivation of an Edible Brown Alga, Cladosiphon okamuranus-I-The Season for Seeding of Zoospore and Its Growth-. Bull Japn Soc Sci Fish 1974, 40(9): 895-902. [In Japanese]

2. Pereira MS, Mulloy B, Maurao PAS: Structure and anticoagulant activity of sulfated fucans. J Biolo Chem 1999, 274(12); 7656-7667.

3. Uehara M, Tako M, Kawashima Y, Fukunaga T, Sho H, Chinen I, Hongo F: Effects of Fucoidan Extracted from Okinawamozuku (Cladosiphon okamuranus TOKIDA) on the Serum Cholesterol Levels in Cholesterol-fed Rats. Oyo Toshitsu Kagaku 1996, 43(2): 149-153. [In Japanese]

4. Araya N, Takahashi K, Sato T, Nakamura T, Sawa C, Hasegawa D, et al.: Fucoidan therapy decreases the proviral load in patients with human T-lymphotropic virus type1-associated neurological disease. Antivir Ther 2011, 16(1): 89-98.

5. Yamamoto Y, Suzuki T, Hirano M, Nagaoka M, Hashimoto S, Shibata H, et al.: Effect of Fucoidan Containing Tea on the Eradication of H. pylori and Non-ulcer Dyspepsia. Jpn Pharmacol Ther 2000, 28(1): 937-942. [In Japanese]

6. Miyoshi M, Abe S, Kasagi T, Hiramatsu K, Ikeda T: Effects of Mozuku-Derived HighMolecular-Weight Fucoidan on lntestinal Motility. J Yonago Med Ass 2013, 64: 69-77. [In Japanese]

7. Nagaoka M, Shibata H, Kimura-Takagi I, Hashimoto S, Kimura K, et al.: Structural study of fucoidan from Cladosiphon okamuranus TOKIDA. Glycoconj J 1999, 16(1): 19-26.

8. Byran GT: The role of urinary tryptophan metabolites in the etiology of bladder cancer. Am J Clin Nutr 1971, 24(7): 841-847.

9. Chung KT, Fulk GE, Slein MW: Tryptophanase of fecal as a possible factor in the etiology of colon cancer. J Natl Cancer Inst 1975, 54(5): 1073-1078.

10. Drasar BS, Hill MJ: Intestinal bacteria and cancer. Am J Clin Nutr 1972, 25(12): 13991404.

11. Mitsuoka T: The world of enterobacteria. Sobunsya, Tokyo; 1980: 43-121.

12. Tomori M, Matsuda T, Nakamura Y, Katena T, Shimothi S, Nagamine T, Ito K: Verification of constipation improvement effect in constipation tenders by beverages containing fucoidan derived from Okinawan mozuku - placebo control randomized double blind crossover comparative test -, Jnp Pharmacol Ther 2016, 44(11): 16211626. [In Japanese]

13. Iraha A, Chinen H, Hokama A, Yonashiro T, Kinjo T, Kishimoto K, et al.: Fucoidan enhances intestinal barrier function by upregulating the expression of claudin-1. World J Gastroenterol 2013, 19(33): 5500-5507. 